\title{
A Note on Local Receive Channel Scheduling versus Transmit Channel Scheduling in Wireless Multi-Channel Ad Hoc Networks
}

\author{
Jens P. Elsner, Ralph Tanbourgi and Friedrich K. Jondral \\ Karlsruhe Institute of Technology, Germany \{jens.elsner, ralph.tanbourgi, friedrich.jondral\}@kit.edu
}

\begin{abstract}
Two strategies for FDMA channel assignment in multi-channel ad hoc networks, where transmissions occur over a maximum distance $r_{\max }$, are compared: local transmit channel and local receive channel FDMA orthogonalization. While transmit scheduling yields higher gains, it also requires a contention resolution mechanism on the MAC layer. Receive scheduling does not necessarily require contention resolution, but performs worse in the relevant low outage regime.
\end{abstract}

\section{INTRODUCTION}

A wireless ad hoc network consists of a collection of nodes communicating without an a-priori known traffic pattern. In contrast to cellular networks, nodes in an ad hoc network must organize network topology and exchange information concerning the network state.

The interrelation of many components of ad hoc networks such as medium access control (MAC), routing, topology control, security, energy expenditure and their interdependencies are still not fully understood and are subject to research. In particular, an appropriate description of the capacity of ad hoc networks has not been found yet as traditional information theoretic approaches fail. A recently proposed structurally simple method for analyzing ad hoc networks with stochastic medium access is the transmission capacity framework [1]. Transmission capacity is defined as the number of successful transmissions taking place simultaneously subject to a constraint on outage probability.

In frequency agile multi-channel networks, the operating bandwidth of the network exceeds the system bandwidth of a single node, allowing for an FDMA component. In such networks, local frequency planning can greatly improve the performance - as measured e.g. by the transmission capacity - of wireless multi-channel ad hoc networks by minimizing interference [2].

In this paper we extend previous work, cf. [1], [2], [3] and references therein, to compare two different approaches to achieve local FDMA scheduling in ad hoc networks: transmit channel scheduling and receive channel scheduling.

The paper is structured as follows. In Section II, the system model is introduced. Section III cites results from [2] for reference, while Section IV offers a comparison to receive channel scheduling. Section V concludes.

\section{SYSTEM MODEL}

We consider an infinitely large set of nodes independently and uniformly distributed in the plane $\mathbb{R}^{2}$ forming a wireless ad hoc network. The transmitter locations are modeled by a stationary Poisson point process (PPP) $\Pi(\lambda)$, where $\lambda$ is the parameter of the PPP and represents the density of transmitters. $X_{i}$ denotes transmitter $i$ as well as its location. The active transmitters give rise to an interference field which, due to its homogeneous nature, can be characterized by a reference connection.

According to Slivnyak's theorem [4], the addition of a specific point does not affect the distribution of $\Pi(\lambda)$. Hence, we can place a reference receiver in the origin and a reference transmitter at a distance of $r$ meters away. The distance $r$ characterizes the maximum distance for which the transmission rate $R$ should be achieved. In the following, the PPP $\Pi(\lambda)$ is considered from the viewpoint of the reference receiver.

Only networks limited by self interference are considered, so we neglect thermal noise and background interference. All transmitters are assumed to transmit with unit power, since in interference-limited wireless networks, the performance of a point-to-point link depends only on the relative signal strengths. Furthermore, interference at the receiver is treated as additive white Gaussian noise and the total operating bandwidth available for transmissions in the network is $B \mathrm{~Hz}$.

This bandwidth is split into $M$ orthogonal channels of bandwidth $B_{m}=\frac{B}{M}$.

We assume a path loss function of the form $\|\vec{x}-\vec{y}\|^{-\alpha}$, where $\alpha>2$ is the path loss exponent. Then, outage probability is defined as [1]

$q(\lambda) \triangleq \mathbb{P}\left\{\frac{r^{-\alpha}}{\sum_{i \in \Pi(\lambda)}\left|X_{i}\right|^{-\alpha}}<\beta\right\}=\mathbb{P}\left\{Y(\lambda)>r^{-\alpha} \beta^{-1}\right\}$,

where $Y(\lambda)=\sum_{i \in \Pi(\lambda)}\left|X_{i}\right|^{-\alpha}$ is the aggregate interference power at the reference receiver and $\beta=2^{\frac{R}{B_{m}}}-1$ is the required signal-to-interference ratio (SIR) threshold for supporting a rate $R$. The corresponding transmission capacity per channel is then given as

$$
c_{m}(\epsilon) \triangleq q^{-1}(\epsilon)(1-\epsilon),
$$

for a target outage probability $\epsilon \in(0,1) \cdot q^{-1}(\epsilon)$ denotes the inverse of the quantile function with respect to $\lambda$, i.e., the 


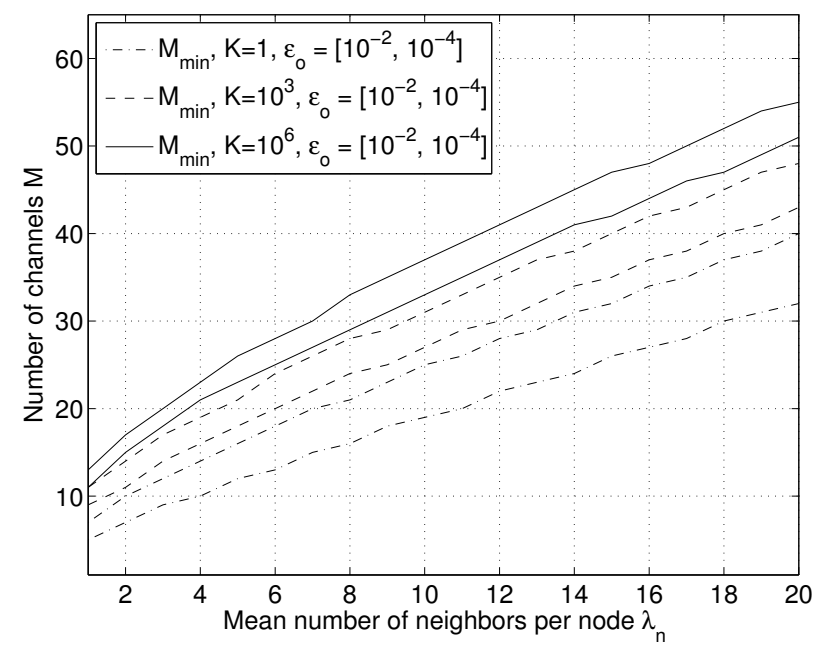

Fig. 1. Number of channels required to allow orthogonalization in a network of $K$ nodes with probability $\epsilon_{o}$.

maximum allowable transmitter density for a target outage probability $\epsilon$. The transmission capacity is a measure of the spatial goodput associated with a given outage probability. The total transmission capacity is the sum of all $M$ channel transmission capacities. Transmissions of nodes are slotted and synchronized.

\section{TRANSMIT SCHEDULING}

The goal of local FDMA transmit scheduling is to assign orthogonal transmit channels to neighboring nodes, i.e., to all nodes within its transmission range $r_{\max }$ to minimize interference.

We will analyze the resulting performance at the reference receiver in the following. However, we have to first ensure that network-wide orthogonalization is feasible in order to make the analysis representative for the whole network. In [2], the minimum number of channels $M_{\min }$ was found for a network with $K$ nodes subject to an orthogonalization failure probability $\epsilon_{o}$, according to

$$
M_{\min } \geq \Phi^{-1}\left(\left(1-\epsilon_{o}\right)^{\frac{1}{K}}, \lambda_{n}\right),
$$

where $\lambda_{n}=\lambda \pi r_{\max }^{2}$ is the average number of transmitters within $r_{\max }$. The term $\Phi^{-1}(z, x)$ is the inverse incomplete Gamma function solving $z=\int_{x}^{\infty} t^{a-1} \exp \{-t\} \mathrm{d} t$ for $a$.

Figure 1 shows the number of channels needed for various $K$ and $\epsilon_{o}$. Even for a large number of nodes $K$ and high network orthogonalization probability $1-\epsilon_{o}$ the required minimum number of channels increases slowly, which reflects a property of the distribution of the maximum of a set of independent Poisson random variables as further examined in [5].

Based on these observations, we derived lower and upper bounds on the success probability at the reference receiver taking into account the probability of successful orthogonal-

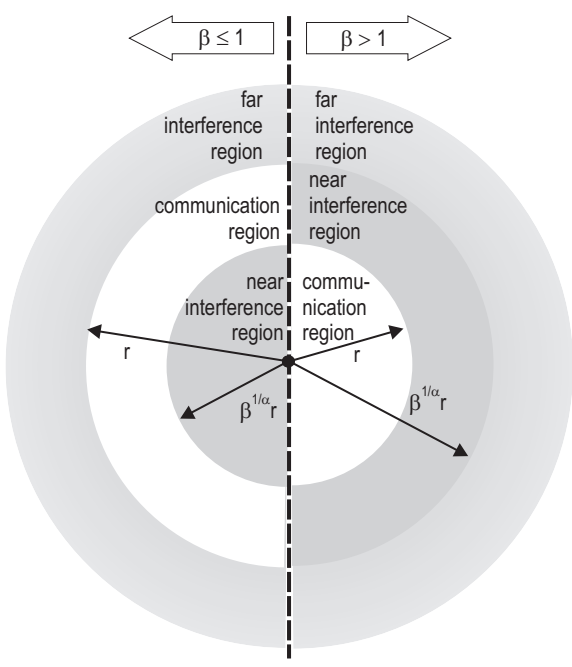

Fig. 2. $\beta \leq 1$ : The communication region is greater or equal to the near interference region; $\beta>1$ : The communication region is smaller than the near interference region. The near interference region hence extends beyond the communication range.

ization at the reference receiver, which is given by

$$
p_{\mathrm{o}}=\sum_{i=0}^{M-1} \exp \left(-\lambda_{n}\right) \frac{\lambda_{n}^{i}}{i !} .
$$

It is assumed that the reference transmission occurrs over the distance $r=r_{\max }$. The bounds are summarized in Table III.

\begin{tabular}{|c||c|c|}
\hline$p_{s}$ & lower bound & upper bound \\
\hline$\beta<1$ & $p_{o}(\lambda)\left(1-\frac{\lambda}{M} \frac{2 \pi r^{2}}{\alpha-2} \beta\right)$ & $\sum_{i=0}^{M-1} e^{-\lambda_{s}} \frac{\lambda_{s}^{2}}{i !}$ \\
$\beta \geq 1$ & $p_{o}(\lambda)\left(1-\frac{\lambda}{M} \frac{2 \pi r^{2} \beta^{\frac{2}{\alpha}}}{\alpha-2}\right) e^{-\frac{\lambda \pi}{M}\left(r_{s}^{2}-r^{2}\right)}$ & $e^{-\frac{\lambda \pi}{M}\left(r_{s}^{2}-r^{2}\right)}$ \\
\hline
\end{tabular}

TABLE I

BOUNDS ON SUCCESS PROBABILITY $p_{s}$.

The terms $r_{s}=r \beta^{\frac{1}{\alpha}}$ and $\lambda_{s}=\lambda \pi r_{s}^{2}$ are the radius and the mean number of transmitters of the near-interference region. Bounds on the transmission capacity (2) can be found by numerically solving $p_{s}(\lambda)$ for $\lambda$. The corresponding transmission capacity results are shown Figure 3.

\section{ReCEIVE Scheduling}

Scheduling as described in Section III requires successful orthogonalization of transmitters before the start of a transmission. This can be achieved by means of a contention resolution protocol but at the cost of additional complexity. Especially in multi-channel networks, this contention resolution poses a challenge, as a node cannot sense every channel. From the assumption of $r \leq r_{\max }$ it follows that receive channel orthogonalization is a means to achieve transmitter orthogonalization: If receiver orthogonalization is applied within $2 r_{\max }$, all transmissions within $r_{\max }$ are guaranteed to be orthogonalized as no two nodes receiving in the same channel are hidden from each other. 


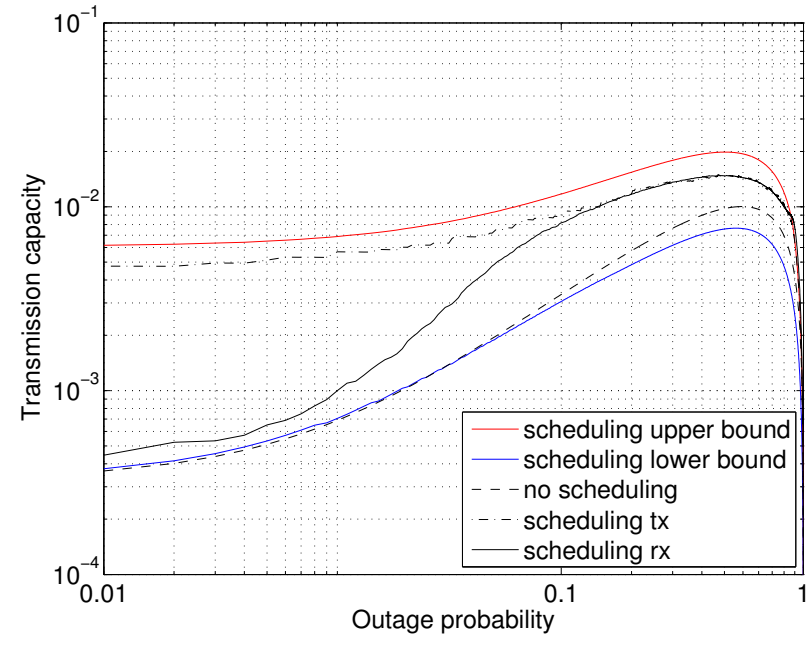

Fig. 3. Transmission capacity for transmit and receive scheduling, $K=$ 1000, $\epsilon_{o}=10^{-2}, R_{m} / B=0.1, \alpha=4$ and $r=10$.

The cost to be paid is the increase in number of channels to achieve network orthogonalization with the same probability $\epsilon_{o}$ : the mean number of nodes increases by a factor of at least 4. The need for more channels naturally leads to a reduction in bandwidth available for point-to-point connections and as such to a reduction in transmission capacity, especially in the - practically most relevant - low outage regime $\epsilon \ll 0.1$.

Figure 3 shows simulation results comparing receive channel scheduling, transmit channel scheduling and no scheduling for the same scheduling distance. As can be seen for the case given, transmit channel scheduling performs better than receive channel scheduling. Both strategies outperform the no scheduling case. At very low outage probabilities, transmit scheduling outperforms receive scheduling significantly (a factor over no scheduling of 13.37 versus 1.67 ), while at high outage probabilities there is no significant difference. This can be explained by the fact that, if node density increases, the number of available channels also increases, making an orthogonalization failure unlikely.

As can be seen, receive scheduling is not particularly effective in the low outage region. As mentioned, a simple way to improve the TC is to orthogonalize receivers within $2 r_{\max }$. From a practical standpoint, there are several approaches which can be used to achieve this:

- using a lower rate data transmission for scheduling (if the same outage probability is required) to schedule at distance $2 r_{\max }$,

- conveying channel assignment over multiple hops,

- or including the implicit information from non-decodable interference when deciding to choose a channel.

The influence of using a lower rate for scheduling will be examined in the following. For successful orthogonalization we have to first ensure that a node is able to exchange information in a robust manner with any other node within distance $2 r_{\max }$. By robust we mean that this information

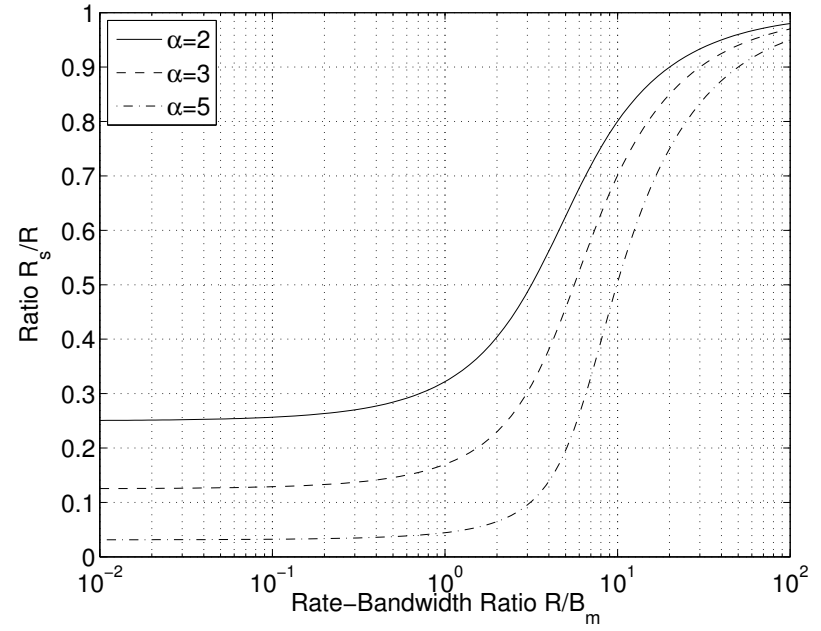

Fig. 4. The ratio $R_{s} / R$ vs. $R_{s} / B_{m}$ for $\alpha=2,3,5$.

exchange must be feasible also in the case of uncoordinated medium access, i.e., "cold start" with slotted Aloha protocol. Using the upper bound on outage probability from [1, (19)] it can be shown that information exchange with nodes within $\gamma r_{\max }$ is successful with probability $1-\epsilon_{s}$ using slotted Aloha, if the rate-bandwidth ratio satisfies

$$
\frac{R_{s}}{B_{m}}=\log _{2}\left(1+\left(\frac{(\alpha-2) \epsilon_{s}}{\lambda \pi \alpha \gamma^{2} r_{\max }^{2}}\right)^{\frac{\alpha}{2}}\right) .
$$

If $\epsilon_{s}=\epsilon$ is required and nodes have overcome the cold start, they can successfully rearrange channel assignment by information exchange with nodes within $2 r_{\max }$ by using local FDMA channel access. The required $R_{s} / B_{m}$ can computed by considering (1) and setting $r^{-\alpha} \beta^{-1} \stackrel{!}{=}(\gamma r)^{-\alpha}\left(2^{-R_{s} / B_{m}}-1\right)$, yielding

$$
\frac{R_{s}}{B_{m}}=\log _{2}\left(\gamma^{-\alpha}\left(2^{\frac{R}{B_{m}}}-1\right)+1\right) .
$$

The resulting ratio $R_{s} / R$ is illustrated in Figure 4 for different $\alpha$. For small $R / B_{m}$, we have that $R_{s} / R \rightarrow \gamma^{-\alpha}$. Note that the result in (6) is not restricted to our local FDMA scheme but applies to general models with path loss attenuation. Thus, we can make the following observations: For low spectral efficiency transmission such as in DS-CDMA, $R_{s} / R$ becomes low as $R$ decreases until convergence at $\gamma^{-\alpha}$. For narrowband transmission (including FH-CDMA), the ratio $R_{s} / R$ increases with $R$ and particularly tends to one as higher modulation is used. These observations are consistent with those presented in [6], where it was shown that FH-CDMA allows for longer hops compared to DS-CDMA at the same rate and for the same outage probability. Here, FH-CDMA is able to support a higher data rate $R_{s}$ at the same range and for the same outage probability.

\section{Discussion AND CONCLUSION}

While transmit scheduling offers the highest transmission capacity in the relevant low outage regime, receive channel 
scheduling can still achieve significant gains without the need for contention resolution before transmission. Although this paper was motivated by the fact that contention resolution is costly, another interesting question is finding the optimum scheduling range in the given model for a given operating outage probability for both scheduling strategies. In future work, we will consider this question and extend the presented results to fading channels.

\section{REFERENCES}

[1] S. Weber, J. Andrews, and N. Jindal, "An overview of the transmission capacity of wireless networks," IEEE Transactions on Communications, Jan 2010, under revision, http://arxiv.org/abs/0809.0016v4.

[2] J. Elsner, R. Tanbourgi, and F. Jondral, "On the Transmission Capacity of Wireless Multi-Channel Ad Hoc Networks with local FDMA scheduling," in International Congress on Ultra Modern Telecommunications and Control Systems, Oct 2010.

[3] R. Tanbourgi, J. Elsner, and F. Jondral, "Lowering Outage Probability in Ad Hoc Networks by Nearest Neighbor FDMA Scheduling," in IEEE WCNC 2011, Cancun, Mexico, Mar 2011, under review.

[4] D. Stoyan, W. Kendall, and J. Mecke, Stochastic geometry and its applications, 2nd ed. Wiley, 1995.

[5] K. Briggs, L. Song, and T. Prellberg, "A note on the distribution of the maximum of a set of poisson random variables," preprint, Mar 2009, http://front.math.ucdavis.edu/0903.4373.

[6] J. Andrews, S. Weber, and M. Haenggi, "Ad hoc networks: To spread or not to spread," IEEE Communications, Dec 2007. 